\title{
True contextuality in a Psychophysical Experiment
}

\author{
Víctor H. Cervantes and Ehtibar N. Dzhafarov
}

\begin{abstract}
Recent crowdsourcing experiments have shown that true contextuality of the kind found in quantum mechanics can also be present in human behavior. In these experiments simple human choices were aggregated over large numbers of respondents, with each respondent dealing with a single context (set of questions asked). In this paper we present experimental evidence of contextuality in individual human behavior, in a psychophysical experiment with repeated presentations of visual stimuli in randomly varying conteXts (arrangements of stimuli). The analysis is based on the Contextuality-by-Default $(\mathrm{CbD})$ theory whose relevant aspects are reviewed in the paper. CbD allows one to detect contextuality in the presence of direct influences, i.e., when responses to the same stimuli have different distributions in different contexts. The experiment presented is also the first one in which contextuality is demonstrated for responses that are not dichotomous, with five options to choose among. CbD requires that random variables representing such responses be dichotomized before they are subjected to contextuality analysis. A theorem says that a system consisting of all possible dichotomizations of responses has to be contextual if these responses violate a certain condition, called nominal dominance. In our experiment nominal dominance was violated in all data sets, with very high statistical reliability established by bootstrapping.
\end{abstract}

KEYWORDS: contextuality, inconsistent connectedness, nominal dominance, psychophysics.

Contextuality (or lack thereof) is a characteristic of a system of random variables. A set of random variables forms a system if each random variable $R_{q}^{c}$ in it is uniquely identified by its content $q$ and its context $c$. The content $q$ is that which the random variable measures or responds to, while the context $c$ is a complex of recorded conditions under which this random variable is observed. As an example, the following set of random variables,

\begin{tabular}{|c|c|c|c||c}
\hline$R_{1}^{1}$ & $R_{2}^{1}$ & & $R_{4}^{1}$ & $c=1$ \\
\hline & $R_{2}^{2}$ & $R_{3}^{2}$ & & $c=2$ \\
\hline$R_{1}^{3}$ & $R_{2}^{3}$ & $R_{3}^{3}$ & & $c=3$ \\
\hline \hline$q=1$ & $q=2$ & $q=3$ & $q=4$ & system $\mathcal{E}$
\end{tabular}

forms a system with three contexts and four contents.

To prevent possible misreadings, we will follow the convention adopted in Dzhafarov and Kujala (2016a) and capitalize the distinguishing letters in the words "conteNt" and "conteXt."

The conteNts could be, e.g., four stimuli (say, questions or light flashes), and conteXts be defined by which two or three of them are presented in a single trial, say, in a fixed succession. Thus, in conteXt $c=1$, three stimuli $(q=1, q=2$, and $q=4)$ are presented, and each of them is being responded to in accordance with some instructions. Depending on the arrangements, a response to a given stimulus can be given immediately after it is presented or after all three of them are presented - such experimental details are immaterial for contextuality analysis insofar as responses 
and stimuli are in a one-to-one correspondence. The responses in the conteXt $c=1$ are the random variables $R_{1}^{1}, R_{2}^{1}, R_{4}^{1}$ shown in the first row of (1). They may be binary (e.g., Yes/No, or I saw it/I did not see it), or they can be multi-valued ones (e.g., each stimulus may have a name, and the task may be to identify which stimulus was shown). The difference between binary and more-than-binary responses plays a central role in the present paper.

Let us explain the intuition behind the notion of contextuality using (1). The random variables within a given conteXt are jointly distributed, and the marginal distribution of a given-conteNt variable may depend on the conteXt in which it is recorded. Thus, the distributions of $R_{2}^{2}$ and $R_{2}^{3}$ may be different, so by knowing the distribution one can guess in which of the two conteXts, $c=2$ or $c=3$, the conteNt $q=2$ is being responded to. This means that the effect of a conteXt upon a distribution is information-carrying, i.e., it is a causal influence. We call such influences direct. The terminology used in physics for direct influences is "signaling," "disturbance," "invasiveness," etc. (Cereceda, 2000; Leggett \& Garg, 1985). In psychology we usually speak of "violations of marginal selectivity" (Dzhafarov, 2003; Dzhafarov \& Kujala, 2016b). If, e.g., the conteNts in (1) are questions, and in each conteXt they are posed in a succession, in the order of their values $(q=1,2,3,4)$, then the response $R_{2}^{2}$ to $q=2$ in conteXt $c=2$ may very well differ in distribution from the response $R_{2}^{3}$ to the same $q=2$ in conteXt $c=3$, because in the later case the respondent could have been affected by the previously asked $q=1$. The (dis)similarity of two conteNt-sharing variables, such as $R_{2}^{2}$ and $R_{2}^{3}$, can be measured by how often their values could coincide had they been jointly distributed (de facto, they are not, because they occur in mutually exclusive conteXts). In other words, the similarity of $R_{2}^{2}$ and $R_{2}^{3}$ is measured by the maximal value of $\operatorname{Pr}\left[T_{2}^{2}=T_{2}^{3}\right]$ among all jointly distributed pairs $\left\{T_{2}^{2}, T_{2}^{3}\right\}$ such that $T_{2}^{2}$ is distributed as $R_{2}^{2}$, and $T_{2}^{3}$ as $R_{2}^{3}$. Any such a pair $\left\{T_{2}^{2}, T_{2}^{3}\right\}$ is called a coupling of $R_{2}^{2}$ and $R_{2}^{3}$, and the couplings with the maximal value of $\operatorname{Pr}\left[T_{2}^{2}=T_{2}^{3}\right]$ are called maximal. We can find maximal couplings for all other conteNt-sharing pairs $\left\{R_{q}^{c}, R_{q}^{c^{\prime}}\right\}$. For some of them we may expect no distributional differences (in our example with questions it could be, e.g., $R_{1}^{1}, R_{1}^{3}$, as in both these cases $q=1$ is asked first), and then the maximal value of $\operatorname{Pr}\left[T_{2}^{2}=T_{2}^{3}\right]$ will be 1 . This is the case of traditional interest in quantum physics. However, generally, both in physics and psychology, differences in distributions of conteNt-sharing random variables should be expected and taken into account. Direct influence is, of course, a form of conteXt-dependence, but it is very different from what is considered contextuality in the proper sense of the word. The latter is detected in the system by showing that the just mentioned maximal couplings of the conteNt-sharing pairs are not compatible with the joint distributions of the random variables within conteXts. In other words, a system is contextual if the joint distributions within conteXts force the conteNt-sharing pairs across conteNts to be more dissimilar than they could be if taken without the conteXts. While direct influences exerted by conteNts are causal (informationcarrying), true contextuality is of a correlational, non-causal nature ${ }^{1}$ More rigorous definitions are given below, in Section 1.

To provide historical perspective, contextuality (without using this term at first) was introduced

\footnotetext{
${ }^{1}$ To prevent objections, direct influences are defined in our theory as the differences in distributions, so one cannot speak of "hidden" influences (Filk, 2015, 2016). Thus, if the variables in system $\mathcal{E}$ are binary, $+1 /-1$, and $\operatorname{Pr}\left[R_{1}^{1}=1\right]=\operatorname{Pr}\left[R_{1}^{3}=1\right]=0.5$, one can imagine that "in reality" conteXt $c=3$ somehow acts upon the "potential values" of $R_{1}^{3}$ reversing their signs, $R_{1}^{3} \rightarrow-R_{1}^{3}$, without changing the distribution. However, this is not considered a "direct influence," because in the given system of random variables these unnoticeable changes do not carry information. If one can actually observe the changes $R_{1}^{3} \rightarrow-R_{1}^{3}$, the system of random variables one deals with changes dramatically, and the CbD analysis then changes accordingly (Dzhafarov, Cervantes, \& Kujala, 2017; Dzhafarov \& Kon, 2018; Dzhafarov \& Kujala, 2018).
} 
in quantum physics by Bell $(1964,1966)$ and Kochen and Specker (1967). They demonstrated that one could meaningfully address, using only observable measurements, the question famously discussed in Bohr's (1935) critique of Einstein, Podolsky, and Rosen (1935). The question is whether all measurement outcomes in a system of measurements can be presented as being determined by some "hidden" random variable in a conteXt-independent way, i.e., using conteXt-independent mappings from the values of this hidden variable into the values of the observed measurement outcomes. With the work of Fine (1982a, b) and Suppes and Zanotti (1981), it became clear that contextuality can also be formulated in terms of the (non)existence of certain joint distributions involving random variables recorded in different conteXts. Although some researchers disagree (Griffiths, 2017), this seems to have become a common way of understanding contextuality (Abramsky, Barbosa, Kishida, Lal, \& Mansfield, 2015; Abramsky, \& Brandenburger, 2011; Araújo, Quintino, Budroni, Cunha, \& Cabello, 2013; Budroni, 2016; Cabello, 2013; Khrennikov, 2008; Klyachko, Can, Binicioglu, \& Shumovsky, 2008; Kurzynski, Ramanathan, \& Kaszlikowski, 2012; Liang, Spekkens, \& Wiseman, 2011; Ramanathan, Soeda, Kurzynski, \& Kaszlikowski, 2012). Probabilistic underpinnings of this understanding have been critically examined by Khrennikov (2000a, b; 2001) and Dzhafarov and Kujala (2016a; 2017a). Irrespective of the debated issues and disagreements, however, contextuality analysis has been moved from physics to probability theory, making it apparent that random variables in contextuality analysis need not represent quantum measurements, they can also be, e.g., responses of biological organisms to stimuli. However, the search for contextuality in psychology was frustrated by the fact that all behavioral systems of random variables exhibit strong direct influences, whereas the theory of contextuality in quantum mechanics, until recently, was only developed for consistently connected systems, those in which conteNt-sharing random variables have identical distributions. When direct influences are taken into account, a large body of experimental data collected in search of contextuality can be shown to exhibit no contextuality (Dzhafarov, \& Kujala, 2014; Dzhafarov, Kujala, Cervantes, Zhang, \& Jones, 2016; Dzhafarov, Zhang, \& Kujala, 2015). Nevertheless two very recent series of experiments unequivocally demonstrate that behavioral data (simple conjoint choices made by people) can be represented by contextual systems of random variables (Basieva, Cervantes, Dzhafarov, \& Khrennikov, in press; Cervantes \& Dzhafarov, 2018). These experiments dealt with responses aggregated over large pools of people, with each person making choices within a single conteXt.

This paper presents the first experimental evidence of contextuality in individual human behavior. In the experiment presented below, each of the three participants made repeated choices in a series of randomized conteXts. A similar experiment, with essentially the same stimuli and similar instructions, has been conducted before, and analyzed in two different ways (Cervantes \& Dzhafarov, 2017a, b): both these analyses revealed no contextuality in the data. The main difference of that experiment from the present one is that in the former all choices were binary, whereas in the present experiment each choice was made among five options. This is an important difference in the theory presented below.

\section{Contextuality-by-Default Theory}

\subsection{Generalities}

A system of random variables is defined as a set of double-indexed random variables

$$
\mathcal{R}=\left\{R_{q}^{c}: c \in C, q \in Q, q \prec c\right\}
$$


where $C$ is a set of conteXts, $Q$ is a set of conteNts, and $q \prec c$ (or $c \succ q$ ) is read "conteNt $q$ is recorded in conteXt $c$ " ${ }^{2}$ Examples of a conteNt $q$ (the "thing" being measured or responded to) are particle's spin in a given direction in a Hilbert space, or a question asked of a person. Examples of a conteXt $c$ may be subsets of conteNts measured "together" (simultaneously or sequentially), or different conditions associated with a given subset of conteNts (e.g., the order in which two fixed questions are asked). The corresponding $R_{q}^{c}$ would then be the spin value (say, "up" or "down") along axis $q$ in a given set $c$ of measured properties, or the response (say, "yes" or "no") to question $q$ asked before or after another question, $q^{\prime}$, with $c=\left(q^{\prime}, q\right)$. As a random variable, $R_{q}^{c}$ is a measurable function from a probability space $\left(X^{c}, \Xi^{c}, \pi^{c}\right)$ to a measurable space $\left(Y_{q}, \Upsilon_{q}\right)$, with the usual meaning of the components. The probability space $\left(Y_{q}, \Upsilon_{q}, p_{q}^{c}\right)$ induced by this function is the distribution of $R_{q}^{c}$. The indices show that $\left(X^{c}, \Xi^{c}, \pi^{c}\right)$ is common to all $R_{q}^{c}$ within a conteXt $c$, i.e., all such $R_{q}^{c}$ are jointly distributed, reflecting the fact that their realizations are empirically linked. Put differently, for any $c \in C$, the set

$$
R^{c}=\left\{R_{q}^{c}: q \in Q, q \prec c\right\}
$$

can be viewed as a random variable. It is a principle of $\mathrm{CbD}$ that any $R_{q}^{c}, R_{q^{\prime}}^{c^{\prime}}$ with $c \neq c^{\prime}$ are stochastically unrelated, i.e., $\left(X^{c}, \Xi^{c}, \pi^{c}\right) \neq\left(X^{c^{\prime}}, \Xi^{c^{\prime}}, \pi^{c^{\prime}}\right)$, reflecting the fact that conteXts are mutually exclusive, so no pairing of the values of $R_{q}^{c}$ and $R_{q^{\prime}}^{c^{\prime}}$ is defined. In particular, the variables in

$$
\mathcal{R}_{q}=\left\{R_{q}^{c}: c \in C, q \prec c\right\}
$$

for a given $q$ are not jointly distributed. However, the distributions of any $R_{q}^{c}, R_{q}^{c^{\prime}}$ in $\mathcal{R}_{q}$ always share the same measurable space, $\left(Y_{q}, \Upsilon_{q}\right)$, reflecting the fact that $R_{q}^{c}$ and $R_{q}^{c^{\prime}}$ have the same conteNt (i.e., they measure or respond to the same "thing").

The next definition is a modification of the usual one (Thorisson, 2000), to better suit our purposes. A (probabilistic) coupling of an indexed set of random variables $\left\{V_{i}\right\}_{i \in I}$ is an identically indexed set of jointly distributed random variables $\left\{W_{i}\right\}_{i \in I}$ such that, for any subset $I^{\prime} \subseteq I$, if the elements of $\left\{V_{i}\right\}_{i \in I^{\prime}}$ are jointly distributed, then $\left\{W_{i}\right\}_{i \in I^{\prime}} \stackrel{\text { dist }}{=}\left\{V_{i}\right\}_{i \in I^{\prime}}$ (the same distribution). In particular, a coupling of a system $\mathcal{R}$ in 2 is a set

$$
S=\left\{S_{q}^{c}: c \in C, q \in Q, q \prec c\right\}
$$

of jointly distributed random variables, such that, for all $c \in C$,

$$
R^{c}=\left\{R_{q}^{c}: q \in Q, q \prec c\right\} \stackrel{\text { dist }}{=}\left\{S_{q}^{c}: q \in Q, q \prec c\right\}=S^{c} .
$$

Returning to our example (1), the following matrix of jointly distributed random variables (or simply, the following random variable) $E$,

\begin{tabular}{|c|c|c|c||c}
\hline$S_{1}^{1}$ & $S_{2}^{1}$ & & $S_{4}^{1}$ & $c=1$ \\
\hline & $S_{2}^{2}$ & $S_{3}^{2}$ & & $c=2$ \\
\hline$S_{1}^{3}$ & $S_{2}^{3}$ & $S_{3}^{3}$ & & $c=3$ \\
\hline \hline$q=1$ & $q=2$ & $q=3$ & $q=4$ & coupling $E$
\end{tabular}

\footnotetext{
${ }^{2}$ Here and throughout, we conveniently confuse $R_{q}^{c}$ and $\left(R_{q}^{c}, c, q\right)$, so that, e.g., $\left\{R_{q}^{c}, R_{q^{\prime}}^{c}\right\}$ consists of two random variables even if $R_{q}^{c} \equiv R_{q^{\prime}}^{c}$, the same measurable function. Also, we follow the common tradition of conveniently confusing functions $R_{q}^{c}$ with their values.
} 
is a coupling of $\mathcal{E}$ if $S^{c} \stackrel{\text { dist }}{=} R^{c}$ for $c=1,2,3$.

Let $\mathrm{P}_{\max }$ be the following statement, well-defined (in the sense of being true or false) for any two jointly distributed random variables $A, B$ :

$$
\mathrm{P}_{\max }(A, B)=\text { "Pr }[A=B] \text { is maximal possible, given the distributions of } A \text { and } B . "
$$

If a coupling $\left\{S_{q}^{c}, S_{q}^{c^{\prime}}\right\}$ of two conteNt-sharing random variables $R_{q}^{c}$ and $R_{q}^{c^{\prime}}$ satisfies this statement, it is called a maximal coupling of $R_{q}^{c}$ and $R_{q}^{c^{\prime}}$. The system $\mathcal{R}$ is noncontextual if $\mathcal{R}$ has a coupling $S$ in which any $\left\{S_{q}^{c}, S_{q}^{c^{\prime}}\right\}$ is a maximal coupling of $R_{q}^{c}$ and $R_{q}^{c^{\prime}}$. Otherwise, if such a coupling $S$ does not exist, the system is contextual. Using our example in (7), system $\mathcal{E}$ is noncontextual if and only if among all its couplings $E$ one can find at least one in which all equalities $S_{1}^{1}=S_{1}^{3}, S_{2}^{1}=S_{2}^{2}$, $S_{2}^{2}=S_{2}^{3}$, and $S_{3}^{2}=S_{3}^{3}$ occur with the maximal probability allowed by their individual distributions. Thus, if $R_{1}^{1}$ and $R_{1}^{3}$ are dichotomous, $+1 /-1$, with $\operatorname{Pr}\left[R_{1}^{1}=1\right]=p$ and $\operatorname{Pr}\left[R_{1}^{3}=1\right]=q$, then the maximal possible probability of $S_{1}^{1}=S_{1}^{3}$ is $1-|p-q|$. Obviously, any subsystem of a noncontextual system (obtained by deleting some of the random variables) is noncontextual, or, equivalently, any system with a contextual subsystem is contextual.

\subsection{Dichotomous random variables}

Most systems of traditional interest consist of dichotomous random variables. Among basic properties of such systems one should mention the following (Dzhafarov, 2017; Dzhafarov, Cervantes, \& Kujala, 2017; Dzhafarov \& Kujala, 2017a, b).

(P1) Adding to or removing from a system a deterministic random variable (attaining a single value with probability 1 ), or a variable that does not share its conteXt or its conteNt with other variables, does not change the system's (non)contextuality (in fact, does not change the degree of contextuality, but we do not discuss this notion here).

(P2) A set of conteNt-sharing random variables $\mathcal{R}_{q}=\left\{R_{q}^{c}: c \in C, q \prec c\right\}$ always has a unique coupling such that any two of its elements satisfy $\mathrm{P}_{\max }$. (Such a coupling is referred to as a multimaximal coupling).

(P3) $T_{q}=\left\{T_{q}^{c}: c \in C, q \prec c\right\}$ is a multimaximal coupling of $\mathcal{R}_{q}$ if and only if, for any $\left\{c_{1}, \ldots, c_{k}\right\} \subseteq$ $C$, the probability of $T_{q}^{c_{1}}=\ldots=T_{q}^{c_{k}}$ is maximal among all couplings of $\left\{R_{q}^{c_{1}}, \ldots, R_{q}^{c_{k}}\right\}$.

(P4) If $\mathcal{R}_{q}=\left\{R_{q}^{c_{1}}, \ldots, R_{q}^{c_{l}}\right\}$ is enumerated so that $\operatorname{Pr}\left[R_{q}^{c_{1}}=1\right] \leq \ldots \leq \operatorname{Pr}\left[R_{q}^{c_{l}}=1\right]$, then $T_{q}$ is a multimaximal coupling of $\mathcal{R}_{q}$ if and only if $\operatorname{Pr}\left[T_{q}^{c_{i}}=T_{q}^{c_{i+1}}\right]$ is maximal for $i=1, \ldots, l-1$ among all possible couplings of $\mathcal{R}_{q}$.

Especially important in quantum-mechanical applications are cyclic systems of ranks $n=2,3, \ldots$. Denoting by $\oplus 1$ cyclic clockwise shift $1 \mapsto 2, \ldots, n-1 \mapsto n, n \mapsto 1$ (and by $\ominus 1$ the opposite shift), a cyclic system of rank $n$ has conteXts $c=1, \ldots, n$, conteNts $q=1, \ldots, n$, and consists of dichotomous $(+1 /-1)$ random variables $\left\{R_{i}^{i}, R_{i \oplus 1}^{i}: i=1, \ldots, n\right\}$. Some examples of such systems are: for $n=2$, question order effects (Wang \& Busemeyer, 2013; Wang, Solloway, Shiffrin, \& Busemeyer, 2014); for $n=3$, the Suppes-Zanotti (Suppes \& Zanotti, 1981), original Bell (1964), and LeggettGarg (Leggett \& Garg, 1985) systems in quantum mechanics, and simple decision making systems in cognition (Asano, Hashimoto, Khrennikov, Ohya, \& Tanaka, 2014; Basieva et al., in press); 
for $n=4$, the EPR/Bohm-Bell-CHSH systems (Bell, 1966; Bohm \& Aharonov, 1957; Clauser \& Horne, 1974; Clauser, Horne, Shimony, \& Holt, 1969; Fine, 1982a, b), and decision making and psychophysical systems (Bruza, Kitto, Nelson, \& McEvoy, 2009; Bruza, Kitto, Ramm, \& Sitbon, 2015; Cervantes \& Dzhafarov, 2017, 2018); for $n=5$, the KCBS system (Klyachko et al., 2008; Lapkiewicz, Li, Schaeff, Langford, Ramelow, Wieśniak, \& Zeilinger, 2011); for $n>5$, some psychophysical systems (Zhang \& Dzhafarov, 2016). The main theoretical result here is

Theorem 1.1 (Kujala \& Dzhafarov, 2016). A cyclic system of rank $n$ is contextual if and only if (denoting expected value by $\langle\cdot\rangle$ )

$$
\max _{\iota_{1}, \ldots, \iota_{k} \in\{-1,1\}, \prod_{i=1}^{n} \iota_{i}=-1} \sum_{i=1}^{n} \iota_{i}\left\langle R_{i}^{i} R_{i \oplus 1}^{i}\right\rangle-(n-2)-\sum_{i=1}^{n}\left|\left\langle R_{i}^{i}\right\rangle-\left\langle R_{i}^{i \ominus 1}\right\rangle\right|>0 .
$$

[] Prior to Kujala and Dzhafarov (2016), this general result was conjectured and proved for small values of $n$ (Dzhafarov, Kujala, \& Larsson, 2015; Kujala \& Dzhafarov, 2015). The special case of this result for consistently connected systems had been proved, by very different means, in (Araújo et al., 2013).

We do not have analogous closed-form criteria for non-cyclic systems, but the theory here is well-developed. There is a general linear programming method for establishing contextuality or lack thereof in any given system with finite sets $C$ and $Q$ and dichotomous random variables (Dzhafarov \& Kujala, 2016a; Dzhafarov, Cervantes, \& Kujala, 2017) (in fact, the method would work for any categorical random variables, but the $\mathrm{CbD}$ approach does not require this, see Section 1.3). The problem is reduced to a certain underdetermined system of linear equations,

$$
\mathbf{M Q}=\mathbf{P}
$$

Here, $\mathbf{P}=(1, \# . ., \ldots 2$.$) , where \# 1$ denotes all probabilities characterizing the distributions within the conteXts (e.g., $\operatorname{Pr}\left[R_{1}^{1}=1, R_{2}^{1}=1, R_{3}^{1}=-1\right]$ ), and $\# 2$ denotes all probabilities characterizing the

maximal couplings $\left\{T_{q}^{c}, T_{q}^{c^{\prime}}\right\}$ of the separate conteNt-sharing pairs (e.g., $\operatorname{Pr}\left[T_{2}^{1}=1, T_{2}^{2}=1\right]$ ); $\mathbf{Q}$ is a vector of probabilities (summing to 1 ) for all possible values of the hypothetical coupling $S$; and $\mathbf{M}$ is a Boolean matrix with 1's in each row corresponding to values of $S$ comprising the events whose probabilities are given in $\mathbf{P}$. The system is noncontextual if and only if these linear equations have a solution for $\mathbf{Q}$ with nonnegative components. The linear programming representation of $\mathrm{CbD}$ naturally leads to its geometric representations by polytopes and graph-theoretic renderings. A detailed version of the latter was recently proposed by Amaral, Duarte, and Oliveira (2018).

\subsection{Arbitrary random variables}

The current version of CbD (Dzhafarov, Cervantes, \& Kujala, 2017; Dzhafarov \& Kujala, 2017a, b) posits that all random variables in a system should be dichotomized before they are submitted to contextuality analysis. One reason for this is that the property $\mathbf{P 2}$ in the previous section does not hold for non-dichotomous variables: a multimaximal coupling need not exist, and when it does, need not be unique. The other reason is that one expects a noncontextual systems to remain noncontextual if some values of a random variable are "lumped together" (e.g., if in $\{1,2,3,4\}$ one ceases to distinguish 1 and 2) (Dzhafarov, Cervantes, \& Kujala, 2017). Dichotomizations are easy 
if in the initial description of an empirical domain all random variables are categorical (i.e., have unordered finite sets of values). One then is interested in all possible dichotomizations: an $n$-valued random variable is replaced with $2^{n-1}-1$ distinct dichotomizations (with unordered pairs of values). For instance, if an initial $R$ has values $\{1,2,3,4\}$, in contextual analysis it is replaced with 7 jointly distributed

$$
\begin{array}{cc|c|c|c|c|c|c|} 
& R_{(1)} & R_{(2)} & R_{(3)} & R_{(4)} & R_{(5)} & R_{(6)} & R_{(7)} \\
\text { values: } \quad 1 \| 2,3,4 & 2 \| 1,3,4 & 3 \| 1,2,4 & 4 \| 1,2,3 & 1,2 \| 3,4 & 1,3 \| 2,4 & 1,4 \| 2,3
\end{array}
$$

Assume, e.g., that in system $\mathcal{E}$ of (1) the variables for $q=1$ have 4 values, variables for $q=3$ have 3 values, and the other two variables are binary. Dichotomization of the system then transforms it into

\begin{tabular}{|c|c|c|c|c|c|c|c|c|c|c|c|c}
\hline$R_{1(1)}^{1}$ & $R_{1(2)}^{1}$ & $R_{1(3)}^{1}$ & $R_{1(4)}^{1}$ & $R_{1(5)}^{1}$ & $R_{1(6)}^{1}$ & $R_{1(7)}^{1}$ & $R_{2}^{1}$ & & & & $R_{4}^{1}$ & $c=1$ \\
\hline & & & & & & & $R_{2}^{2}$ & $R_{3(1)}^{2}$ & $R_{3(2)}^{2}$ & $R_{3(3)}^{2}$ & & $c=2$ \\
\hline$R_{1(1)}^{3}$ & $R_{1(2)}^{3}$ & $R_{1(3)}^{3}$ & $R_{1(4)}^{3}$ & $R_{1(5)}^{3}$ & $R_{1(6)}^{3}$ & $R_{1(7)}^{3}$ & $R_{2}^{3}$ & $R_{3(1)}^{3}$ & $R_{3(2)}^{3}$ & $R_{3(3)}^{3}$ & & $c=3$ \\
\hline \hline$q=1(1)$ & $1(2)$ & $1(3)$ & $1(4)$ & $1(5)$ & $1(6)$ & $1(7)$ & 2 & $3(1)$ & $3(2)$ & $3(3)$ & 4 & system $\mathcal{E}^{*}$ \\
\hline
\end{tabular}

where the numbers in parentheses encode different dichotomizations. The procedure effectively splits old conteNts into new conteNts. The size of the system increases only in visual appearance, because in each row of $\mathcal{E}^{*}$ the support of the joint distribution is precisely the same as in system $\mathcal{E}$. The original system is considered contextual if its dichotomization is contextual. The main result here is

Theorem (Dzhafarov, Cervantes, \& Kujala, 2017). A system of categorical random variables (before dichotomization) is contextual if, for some $\left(q, c, c^{\prime}\right)$, neither of $R_{q}^{c}, R_{q}^{c^{\prime}}$ nominally dominates the other.

It is this theorem that we use to analyze the experiment below. The meaning of nominal dominance is as follows: given $A$ and $B$ with the same set of values $\{1, \ldots, k\}, A$ nominally dominates $B$ if the inequality $\operatorname{Pr}[A=i]<\operatorname{Pr}[B=i]$ holds for no more than one value of $i=1, \ldots, k$ (i.e., if $\operatorname{Pr}[A=i] \geq \operatorname{Pr}[B=i]$ for at least $k-1$ of them). Thus, among the pairs of probability distributions below,

\begin{tabular}{r|c|c|c|c|c|}
$(\mathrm{i}) \quad$ Values & 1 & 2 & 3 & 4 & 5 \\
\hline probabilities for $A$ : & 0.1 & 0.2 & 0.2 & 0.5 & 0 \\
probabilities for $B$ : & 0.1 & 0.2 & 0.2 & 0.5 & 0
\end{tabular}

\begin{tabular}{r|c|c|c|c|c|} 
(ii) & 1 & 2 & 3 & 4 & 5 \\
\hline probabilities for $A$ : & 0.1 & 0.2 & 0.2 & 0.5 & 0 \\
probabilities for $B$ : & 0.2 & 0.1 & 0.2 & 0.5 & 0
\end{tabular} ,

\begin{tabular}{r|c|c|c|c|c|} 
(iii) Values & 1 & 2 & 3 & 4 & 5 \\
\hline probabilities for $A$ : & 0.1 & 0.2 & 0.2 & 0.5 & 0 \\
probabilities for $B$ : & 0.5 & 0 & 0.1 & 0.4 & 0
\end{tabular} ,

\begin{tabular}{r|c|c|c|c|c|} 
(iv) Values & 1 & 2 & 3 & 4 & 5 \\
\hline probabilities for $A$ : & 0.1 & 0.2 & 0.2 & 0.5 & 0 \\
probabilities for $B:$ & 0.3 & 0.3 & 0.1 & 0.2 & 0.1
\end{tabular} ,

in (i) and (ii) $A$ and $B$ nominally dominate each other, in (iii) $A$ nominally dominates $B$, and in (iv) neither of the two random variables nominally dominates the other.

The theorem above tells us that if we are interested in all possible dichotomizations, we may not need to actually create them to determine that the system is contextual. It suffices instead to find at least one instance when neither of two original (as observed, before dichotomization) 


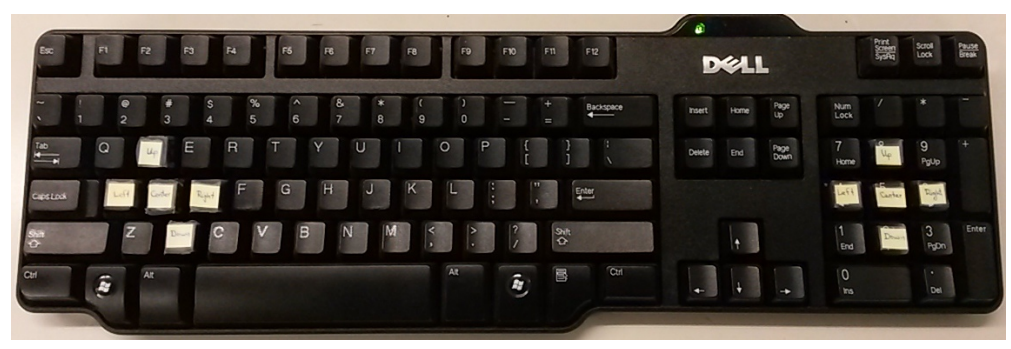

Figure 1: Layout of the keyboard with the response keys stickers for left and right stimuli.

conteNt-sharing random variables nominally dominates the other, as in (iv) above. The condition is only sufficient but not necessary for contextuality: if nominal dominance is found in all pairs of conteNt-sharing random variables, the system may or may not be contextual.

\section{Double-Identification Experiment}

\subsection{Method}

\subsubsection{Participants}

Three volunteers, graduate students at Purdue University, one female and two males (including the first author of this paper), with normal or corrected to normal vision, participated in this study. The experimental program was regulated by Purdue University's IRB protocol \#1202011876. The participants are identified as $\mathrm{P} 1, \mathrm{P} 2$, and $\mathrm{P} 3$ in the text below.

\subsubsection{Equipment}

A personal computer was used with an Intel ${ }_{\circledR}$ Core $^{T M}$ processor running Windows XP, and with a 24-in. monitor with a resolution of $1920 \times 1200$ pixels $(\mathrm{px})$. The participant's head was steadied in a chin-rest with forehead support at $90 \mathrm{~cm}$ distance from the monitor; at this distance a pixel on the screen subtended $62 \mathrm{sec}$ arc. The response keys on a US 104-key keyboard were indicated by stickers with the corresponding response labels (see Figure 1).

\subsubsection{Stimuli}

The stimuli presented on the computer screen consisted of two brightly grey colored circles (RGB 100-100-100) on a black background, with their centers $320 \mathrm{px}$ apart horizontally, each circle having the radius of $135 \mathrm{px}$ and $4 \mathrm{px}$ wide circumference. Each circle contained within it a dot of $4 \mathrm{px}$ in diameter, that could be located in the circle's center or $4 \mathrm{px}$ away from it, in the left, right, upward or downward direction. An example of the stimuli is shown in Figure 2.

\subsubsection{Procedure}

In each trial the participant was asked to indicate, for each circle, whether the dot was in its center or shifted in one of the four directions (up, down, left, or right). The responses were given by pressing in any order and holding together two designated keys, one for each location in each circle, 


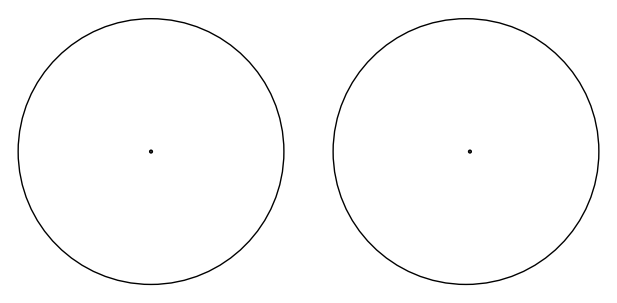

Figure 2: An example of the stimuli in experiment (in reversed contrast and not to scale). In the left circle the dot is in the center, in the right one it is shifted to the right by $4 \mathrm{px}(\simeq 4.1 \mathrm{~min} \operatorname{arc})$. The participant's task was to identify the location of the dot in each of the two circles by pressing corresponding keys on a keyboard.

as shown in Fig. 2. The stimuli were displayed until both keys were pressed. Then, the dots in each circle disappeared, and the next pair of dots appeared $600 \mathrm{~ms}$ later. The circles, with or without the dots, remained displayed continuously throughout the experiment. (Response times were recorded but not used in the data analysis.)

Each participant completed between 20 and 23 experimental sessions, each lasting 30 minutes and consisting of about 380 trials recorded and used for subsequent analysis. The experimental sessions were preceded by two training sessions, excluded from the analysis. The first 75 trials of each training session were practice trials in which the participants received feedback as to whether their response for each of the two circles was correct or not. No feedback was given in the experimental trials.

\subsection{Experimental conteXts and conteNts}

In each of two circles the dot presented could be in one of 5 locations: at the center, or shifted to the left, right, up, or down. These locations formed conteNts of the random variables in the probabilistic description of the experiment, denoted as shown in Table 1. The same table shows that the $5 \times 5$ pairs of locations of the two dots formed 25 conteXts. In each experimental session, all conteXts were presented [close-to-]equal numbers of times (about 15).

For each session, each trial was randomly assigned to one of the conditions in Figure 1. The number of experimental sessions was chosen so that the expected number of experimental trials in each of the conteXts was at least 300. This number of observations was chosen based on Cepeda Cuervo, Aguilar, Cervantes, Corrales, Díaz, and Rodríguez (2008), whose results show that coverage errors with respect to nominal values are below $1 \%$ for most confidence intervals for proportions with $n>300$.

The system of random variables describing the experiment is shown in Figure 3 
Table 1: Notation used for the conteXts and the conteNts: $c, l, r, u$, and $d$ denote that the dot is, respectively, in the center, shifted to the left, to the right, up, or down. The 25 conteXts are denoted $c c, c u, d u$, etc., the left (right) symbol indicating the location of the dot in the left (respectively, right) circle. To denote conteNts, the location of a dot is shown on the left (for the left circle) or on the right (for the right circle) of a dash: thus, $c$ - denotes the dot in the center of the left circle, $-l$ denotes the dot shifted to the left in the right circle, etc.

\begin{tabular}{|c|c|c|c|c|c|c|}
\hline & \multicolumn{5}{|c|}{ Right circle conteNts } \\
\hline & & $(-c)$ & $(-l)$ & $(-r)$ & $(-u)$ & $(-d)$ \\
\hline \multirow{5}{*}{ Left circle conteNts } & Center $(c-)$ & $c c$ & $c l$ & $\mathrm{cr}$ & $c u$ & $c d$ \\
\hline & Left $(l-)$ & $l c$ & $l l$ & $l r$ & $l u$ & $l d$ \\
\hline & Right $(r-)$ & $r c$ & $r l$ & $r r$ & ru & $r d$ \\
\hline & $\mathrm{Up}(u-)$ & $u c$ & $u l$ & $u r$ & $u u$ & $u d$ \\
\hline & Down $(d-)$ & $d c$ & $d l$ & $d r$ & $d u$ & $d d$ \\
\hline
\end{tabular}

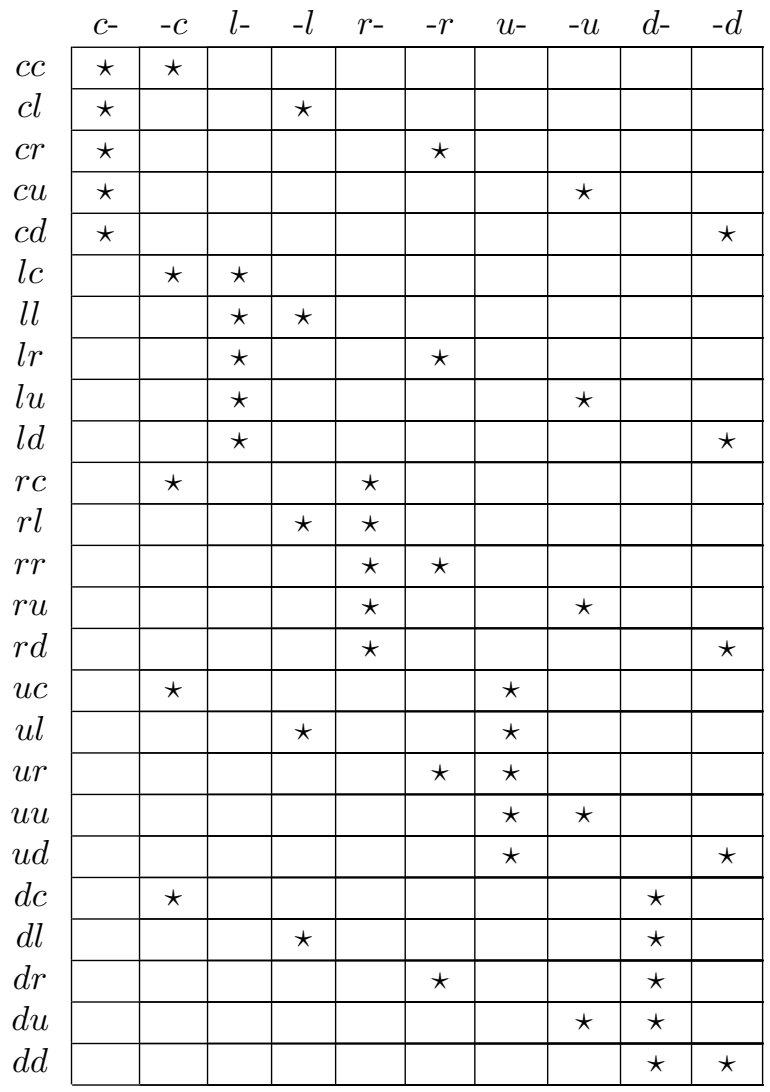

Figure 3: The conteNt-conteXt system of measurements for the double detection experiment. The cell corresponding to conteXt $x y$ and conteNt $z$ (with $z$ being $x$ - or $-y$ ), if it contains a star, represents the random variable $R_{z}^{x y}$; the absence of a star means that conteNt $z$ was not measured in conteXt $x y$. For instance, $x y=c c$ and $z=c$ - define a random variable $R_{c-}^{c c}$. There are two random jointly distributed variables, $R_{x-}^{x y}$ and $R_{-y}^{x y}$, in each conteXt $x y$, and their joint distribution is defined by the probabilities: $\operatorname{Pr}\left[R_{x-}^{x y}=j, R_{-y}^{x y}=k\right]$ where $j, k \in\{$ center, left, right, up, down $\}$. 
Table 2: Bootstrap estimates of the probabilities for the systems to satisfy the nominal dominance condition.

\begin{tabular}{|c|c|c|c|}
\hline ConteNt & P1 & P2 & P3 \\
\hline$c-$ & 0.038 & 0.000 & 0.000 \\
\hline$l$ - & 0.000 & 0.000 & 0.224 \\
\hline$r-$ & 0.000 & 0.000 & 0.003 \\
\hline$u-$ & 0.429 & 0.000 & 0.023 \\
\hline$d-$ & 0.002 & 0.000 & 0.001 \\
\hline$-c$ & 0.412 & 0.000 & 0.000 \\
\hline$-l$ & 0.019 & 0.000 & 0.385 \\
\hline$-r$ & 0.000 & 0.000 & 0.015 \\
\hline$-u$ & 0.566 & 0.001 & 0.034 \\
\hline$-d$ & 0.001 & 0.000 & 0.000 \\
\hline $\begin{array}{c}\text { Overall, } \\
\text { for all contents }\end{array}$ & 0.000 & 0.000 & 0.000 \\
\hline
\end{tabular}

\subsection{Results}

The complete set of results obtained in the experiment (excluding training sessions) is stored in "Contextuality in a psychophysical double-identification experiment", https://doi.org/10.7910/ DVN/FCT9VO. The data used in the analysis of the nominal dominance condition are shown in Tables A1, A2, and A3, placed in Appendix. These tables show the estimated probabilities with which each of the three participants responded in each of five possible ways (center, left, right, up, and down) to the left stimulus and to the right stimulus, in each of the 25 conteXts. For all participants, the nominal dominance condition fails for at least one pair of random variables for each of the conteNts. This means that, for all three participants, the pattern of the results indicates contextuality.

To assess the reliability of these results, we generated 100000 bootstrap resamples for each participant: each bootstrap resample was generated by independently selecting, with replacement, a random sample from (and of the same size as) the responses given in the experiment to each of the two circles in each conteXt. The proportions of resamples in which nominal dominance was observed are presented in Table 2, for each conteNt separately, and (in the bottom row of the table) for all conteNts simultaneously. Note that it is the latter that matters for our analysis: the system may be noncontextual only if nominal dominance is satisfied for all pairs of conteNt-sharing random variables. This was observed for none of the resamples and none of the participants. We can model this situation, for each participant, as a sequence of 100000 binomial trials with zero successes. If $p$ denotes the probability of this happening (let us label this as a "success"), We can model the results, for each participant, as a Bernoulli sequence of length 100000, with probability of a "success" (overall compliance with nominal dominance) being $p$, and the observed number of successes being zero. The exact 99.999\% Clopper-Pearson (Clopper \& Pearson, 1934) confidence interval for $p$ is $[0,0.00012]$. We can clearly dismiss the possibility that our data result from random perturbations of a pattern that satisfies nominal dominance. 


\section{Discussion}

Based on the CbD analysis of many published experiments in none of which contextuality was found, it was tempting to hypothesize that all behavioral systems were noncontextual (Dzhafarov, Kujala, Cervantes, Zhang, \& Jones, 2016; Dzhafarov, Zhang, \& Kujala, 2015; Zhang \& Dzhafarov, 2016). This hypothesis was rejected by recent crowdsourcing experiments (Basieva et al., in press; Cervantes \& Dzhafarov, 2018), but the question remained open as to whether contextuality can also be observed in individual human behavior. In the crowdsourcing experiments the stimuli were questions to be answered in one of two ways. In such an experiment a repeated presentation of a question to the same person cannot be viewed as a repeated recording of the same random variable, because the person would most likely remember her previous answers and repeat them not to contradict herself, or would deliberately vary them due to the phenomenon of satiation. Therefore, to investigate contextuality in a within-subject paradigm, one has to use stimuli that do not have any distinguishing characteristics by which they can be remembered. Thus, if a variety of weak flashes varying in intensity are judged in terms of "I have seen it" or "I have not seen it," there is no way the observer may remember seeing a particular flash before, unless this flash was seen with probability 1. Analogously, in our experiment, there was no way a participant could remember seeing a specific dot position in one of the circles, as no position was identified perfectly.

A previously conducted experiment (Cervantes \& Dzhafarov, 2017a, b), similar to the one presented in this paper, revealed no contextuality, i.e., all conteXt-dependence in it could be attributed to direct influences. In that experiment the dots within two circles could vary on three levels (center, up, down) and the responses were dichotomous: "in the center" or "not in the center." As it turns out, switching to questions with five possible answers (and increasing the number of conteNts to five to match them) changed the system from noncontextual to contextual.

The overall conteXt-dependence in our experiment means that a given location $q$ of the dot in a circle is judged differently for different locations $q^{\prime}$ of the dot in the other circle. This direct influence of $q^{\prime}$ on responses to $q$ manifests itself in the changing distribution of the responses to $q$ as $q^{\prime}$ changes. The contextuality of the system, however, shows that these direct influences cannot account for the entire situation: the changes in the identity of the random variable representing the responses to $q$ in different conteXts are greater than warranted by their distributional differences. This is another way of stating the definition of a contextual system, according to which the joint distributions of the random variables within conteXts force conteNt-sharing random variables (responses to the same $q$ at different $q^{\prime}$ ) to be more dissimilar than warranted by the difference in their distributions.

The relationship between the two forms of conteXt-dependence in a contextual system, direct influences and contextuality proper, is a complex issue of which we have very little knowledge at present. A remarkable fact is that this relationship seems to be different in systems of binary random variables (at least in cyclic systems, mentioned in Section 1.2) and in systems of multivalued random variables. As is evident from $\sqrt{9}$, the direct influences and contextuality in a cyclic system are antagonistic. Direct influences in (9) are represented by

$$
\sum_{i=1}^{n}\left|\left\langle R_{i}^{i}\right\rangle-\left\langle R_{i}^{i \ominus 1}\right\rangle\right|
$$

and as this quantity increases, the value of the left-hand-side expression in (9) decreases, making the system less likely to be contextual. In our present experiment the situation is more complex. 
Direct influences here are responsible for the differences between the distributions

\begin{tabular}{r|c|c|c|c|c|} 
responses to $q$ in context $q q^{\prime}:$ & center & left & right & up & down \\
\hline probabilities: & $p_{1}$ & $p_{2}$ & $p_{3}$ & $p_{4}$ & $p_{5}$
\end{tabular}

and

\begin{tabular}{r|c|c|c|c|c} 
responses to $q$ in context $q q^{\prime \prime}:$ & center & left & right & up & down \\
\hline probabilities: & $p_{1}^{\prime}$ & $p_{2}^{\prime}$ & $p_{3}^{\prime}$ & $p_{4}^{\prime}$ & $p_{5}^{\prime}$
\end{tabular} .

In the absence of all direct influences, i.e., with $p_{i}=p_{i}^{\prime}$ for all $i$, the nominal dominance is trivially satisfied. This does not mean that the system in noncontextual, but its contextuality will have to be established by other means, generally, by solving the linear programming task 10 . Direct influences must be present to break the nominal dominance relation and thereby allow us to establish contextuality "easily." More work is needed to understand this relationship better.

Acknowledgments. This research has been partially supported by AFOSR grant FA9550-14-10318.

\section{References}

Abramsky, S., Barbosa, R. S., Kishida, K., Lal, R., \& Mansfield, S. (2015). Contextuality, cohomology and paradox. Computer Science Logic, 2015, 211-228.

Abramsky, S. \&Brandenburger, A. (2011). The sheaf-theoretic structure of non-locality and contextuality. New Journal of Physics, 13, 113036-113075.

Amaral, B., Duarte, C., \& Oliveira, R. I. (2018). Necessary conditions for extended noncontextuality in general sets of random variables. Journal of Mathematical Physics, 59, 072202.

Araújo, M., Quintino, M. T. , Budroni, C. , Cunha, M. T., \& Cabello, A. (2013). All noncontextuality inequalities for the n-cycle scenario. Physical Review A, 88, 022118.

Asano, M., Hashimoto, T., Khrennikov, A., Ohya, M., \& Tanaka, T. (2014). Violation of contextual generalization of the Leggett-Garg inequality for recognition of ambiguous figures. Physica Scripta, T163, 014006.

Basieva, I., Cervantes, V. H., Dzhafarov, E. N., \& Khrennikov, A. (in press). True contextuality beats direct influences in human decision making. Journal of Experimental Psychology: General (available as arXiv:1807.05684).

Bell, J. (1964). On the Einstein-Podolsky-Rosen paradox. Physics, 1, 195-200.

Bell, J. (1966). On the problem of hidden variables in quantum mechanics. Review of Modern Physics, 38, 447-453.

Bohm, D., \& Aharonov, Y. (1957). Discussion of experimental proof for the paradox of Einstein, Rosen and Podolski. Physical Review, 108, 1070-1076.

Bohr, N. (1935). Can quantum-mechanical description of physical reality be considered complete? Physical Review, 48, 696-702. 
Budroni, C. (2016). Temporal Quantum Correlations and Hidden Variable Models. Springer: Heidelberg.

Budroni, C. \& Emary, C. (2014). Temporal quantum correlations and Leggett-Garg inequalities in multilevel systems. Physical Review Letters, 113, 050401.

Bruza, P. D., Kitto, K., Nelson, D., \& McEvoy, C. (2009). Is there something quantum-like about the human mental lexicon? Journal of Mathematical Psychology, 53, 362-377.

Bruza, P. D., Kitto, K., Ramm, B. J., \& Sitbon, L. (2015). A probabilistic framework for analysing the compositionality of conceptual combinations. Journal of Mathematical Psychology, 67, 26-38.

Cabello, A. (2013). Simple explanation of the quantum violation of a fundamental inequality. Physical Review Letters, 110, 060402.

Cepeda Cuervo, E., Aguilar, W., Cervantes, V. H., Corrales, M., Díaz, I. \& Rodríguez, D. (2008). Intervalos de confianza e intervalos de credibilidad para una proporción. Revista Colombiana de Estadística, 31, 211-228.

Cereceda, J. (2000). Quantum mechanical probabilities and general probabilistic constraints for Einstein-Podolsky-Rosen-Bohm experiments. Foundations of Physics Letters, 13, 427-442.

Cervantes, V. H., \& Dzhafarov, E. N. (2017a). Exploration of contextuality in a psychophysical double-detection experiment. Lecture Notes in Computer Science, 10106, 182-193.

Cervantes, V. H., \& Dzhafarov, E. N. (2017b). Advanced analysis of quantum contextuality in a psychophysical double-detection experiment. Journal of Mathematical Psychology, 79, 77-84.

Cervantes, V. H., \& Dzhafarov, E. N. (2018). Snow Queen is evil and beautiful: Experimental evidence for probabilistic contextuality in human choices. Decision, 5, 193-204.

Clauser, J. F., \& Horne, M. A. (1974). Experimental consequences of objective local theories. Physical Review D, 10, 526-535.

Clauser, J. F. , Horne, M. A. , Shimony, A., \& Holt, R. A. (1969). Proposed experiment to test local hidden-variable theories. Physical Review Letters, 23, 880-884.

Clopper, C. \& Pearson, E. S. (1934). The use of confidence or fiducial limits illustrated in the case of the binomial. Biometrika, 26, 404-413.

Dzhafarov, E. N. (2003). Selective influence through conditional independence. Psychometrika, $68,7-26$.

Dzhafarov, E. N. (2017). Replacing nothing with something special: Contextuality-by-Default and dummy measurements. In A. Khrennikov \& T. Bourama (Eds.). Quantum Foundations, Probability and Information (pp. 39-44). Berlin: Springer.

Dzhafarov, E. N., Cervantes, V. H., \& Kujala, J. V. (2017). Contextuality in canonical systems of random variables. Philosophical Transactions of the Royal Society A, 375, 20160389.

Dzhafarov, E. N., \& Kon, M. (2018). On universality of classical probability with contextually labeled random variables. Journal of Mathematical Psychology, 85, 17-24 
Dzhafarov, E. N., \& Kujala, J. V. (2014). Selective influences, marginal selectivity, and Bell/CHSH inequalities. Topics in Cognitive Science, 6, 121-128.

Dzhafarov, E. N., \& Kujala, J. V. (2016a). Context-content systems of random variables: The contextuality-by-default theory. Journal of Mathematical Psychology, 74, 11-33.

Dzhafarov, E. N., \& Kujala, J. V. (2016b). Probability, random variables, and selectivity. In W. Batchelder, H. Colonius, E. N. Dzhafarov, \& J. Myung (Eds). The New Handbook of Mathematical Psychology (pp. 85-150). Cambridge University Press.

Dzhafarov, E. N. \& Kujala, J. V. (2017a). Probabilistic foundations of contextuality. Fortschritte der Physik, 65, 1-11.

Dzhafarov, E. N. \& Kujala, J. V. (2017b). Contextuality-by-Default 2.0: Systems with binary random variables. Lecture Notes Computer Sciences, 10106, 16-32.

Dzhafarov, E. N., \& Kujala, J. V. (2018). Contextuality analysis of the double slit experiment (with a glimpse into three slits). Entropy, 20, 278.

Dzhafarov, E. N., Kujala, J. V., Cervantes, V. H., Zhang, R., \& Jones, M. (2016). On contextuality in behavioral data. Philosophical Transactions of the Royal Society A, 374, 20150234.

Dzhafarov, E. N., Kujala, J. V., \& Larsson, J.-A. (2015). Contextuality in three types of quantum-mechanical systems. Foundations of Physics, 7, 762-782.

Dzhafarov, E. N., Zhang, R., \& Kujala, J. V. (2015). Is there contextuality in behavioral and social systems? Philosophical Transactions of the Royal Society A, 374, 20150099.

Einstein, A., Podolsky, B., \& Rosen, N. (1935). Can quantum-mechanical description of physical reality be considered complete? Physical Review, 47, 777-780.

Fine, A. (1982a). Hidden variables, joint probability, and the Bell inequalities. Physical Review Letters, 48, 291-295.

Fine, A. (1982b). Joint distributions, quantum correlations, and commuting observables. Journal of Mathematical Physics, 23,1306-1310.

Filk, T. (2015). It is the theory which decides what we can observe. In E. N. Dzhafarov, S. Jordan, R. Zhang, \& V. Cervantes (Eds). Contextuality from Quantum Physics to Psychology (pp. 77-92). New Jersey: World Scientific.

Filk, T. (2016). A mechanical model of a PR-Box. arXiv:1507.06789.

Griffiths, R. B. (2017). What quantum measurements measure. Physical Review A, 96, 032110.

Klyachko, A. A., Can, M. A., Binicioglu, S., \& Shumovsky, A. S. (2008). A simple test for hidden variables in spin-1 system. Physical Review Letters, 101, 020403.

Khrennikov, A. (2000a). Non-Kolmogorov probability models and modified Bell's inequality. Journal of Mathematical Physics, 41, 1768-1777.

Khrennikov, A. (2000b). A perturbation of CHSH inequality induced by fluctuations of ensemble distributions. Journal of Mathematical Physics, 41, 5934-5944.

Khrennikov, A. (2001). Contextualist viewpoint to Greenberger-Horne-Zeilinger paradox. Physical Letters A, 278, 307-314. 
Khrennikov, A. (2008). Bell-Boole inequality: Nonlocality or probabilistic incompatibility of random variables? Entropy, 10, 19-32.

Kochen, S., \& Specker, E. P. (1967). The problem of hidden variables in quantum mechanics. Journal of Mathematics and Mechanics, 17, 59-87.

Kujala, J. V., \& Dzhafarov, E. N. (2015). Probabilistic contextuality in EPR/Bohm-type systems with signaling allowed. In E. N. Dzhafarov, S. Jordan, R. Zhang, \& V. Cervantes (Eds). Contextuality from Quantum Physics to Psychology (pp. 287-308). New Jersey: World Scientific.

Kujala, J. V., \& Dzhafarov, E. N. (2016). Proof of a conjecture on contextuality in cyclic systems with binary variables. Foundations of Physics, 46, 282-299.

Kurzynski, P., Ramanathan, R., \& Kaszlikowski, D. (2012). Entropic test of quantum contextuality. Physical Review Letters, 109, 020404.

Lapkiewicz, R., Li, P., Schaeff, C., Langford, N. K., Ramelow, S., Wieśniak, M., \& Zeilinger, A. (2011). Experimental non-classicality of an indivisible quantum system. Nature, 474, 490-493.

Leggett, A., \& Garg, A. (1985). Quantum mechanics versus macroscopic realism: Is the flux there when nobody looks? Physical Review Letters, 54, 857.

Liang, Y.-C., Spekkens, R. W., \& Wiseman, H. M. (2011). Specker's parable of the overprotective seer: A road to contextuality, nonlocality and complementarity. Physics Reports, 506, 1-39.

Ramanathan, R., Soeda, A., Kurzynski, P., \& Kaszlikowski, D. (2012). Generalized monogamy of contextual inequalities from the no-disturbance principle. Physical Review Letters, 109, 050404.

Suppes, P., \& Zanotti, M. (1981). When are probabilistic explanations possible? Synthese, 48, 191-199.

Thorisson, H. (2000). Coupling, Stationarity, and Regeneration. New York: Springer.

Wang, Z., \& Busemeyer, J. R. (2013). A quantum question order model supported by empirical tests of an a priori and precise prediction. Topics in Cognitive Science, 5, 689-710.

Wang, Z., Solloway, T., Shiffrin, R. M., \& Busemeyer, J. R. (2014). Context effects produced by question orders reveal quantum nature of human judgments. Proceedings of the National Academy of Sciences, 111, 9431-9436.

Zhang, R., \& Dzhafarov, E. N. (2016). Testing contextuality in cyclic psychophysical systems of high ranks. Lecture Notes in Computer Science, 10106, 151-162. 


\section{Appendix: Data tables}

Table A1: Empirical estimates of marginal distributions for the conteNt-conteXt system in Fig. 3 for participant P1.

\begin{tabular}{|c|c|c|c|c|c|c|c|c|c|c|c|}
\hline \multirow{2}{*}{$\begin{array}{l}\mathrm{P} 1 \\
\text { Context }\end{array}$} & \multirow[b]{2}{*}{ Trials } & \multicolumn{5}{|c|}{ Left response } & \multicolumn{5}{|c|}{ Right response } \\
\hline & & Center & Left & Right & Up & Down & Center & Left & Right & $\mathrm{Up}$ & Down \\
\hline$c c$ & 336 & .318 & .521 & .000 & .155 & .006 & .235 & .455 & .000 & .310 & .000 \\
\hline cl & 334 & .213 & .656 & .000 & .132 & .000 & .015 & .871 & .000 & .108 & .006 \\
\hline$c r$ & 336 & .390 & .435 & .000 & .155 & .021 & .601 & .060 & .018 & .318 & .003 \\
\hline$c u$ & 336 & .298 & .554 & .000 & .143 & .006 & .021 & .149 & .000 & .830 & .000 \\
\hline$c d$ & 336 & .265 & .574 & .000 & .152 & .009 & .271 & .613 & .000 & .030 & .086 \\
\hline$l c$ & 334 & .036 & .931 & .000 & .027 & .006 & .195 & .527 & .000 & .278 & .000 \\
\hline$l l$ & 335 & .024 & .928 & .000 & .042 & .006 & .021 & .860 & .000 & .119 & .000 \\
\hline$l r$ & 335 & .051 & .913 & .003 & .030 & .003 & .558 & .122 & .018 & .299 & .003 \\
\hline$l u$ & 335 & .054 & .904 & .000 & .033 & .009 & .042 & .176 & .003 & .779 & .000 \\
\hline$l d$ & 334 & .042 & .910 & .003 & .042 & .003 & .314 & .605 & .000 & .024 & .057 \\
\hline$r c$ & 333 & .763 & .081 & .033 & .117 & .006 & .246 & .483 & .000 & .270 & .000 \\
\hline$r l$ & 334 & .605 & .159 & .051 & .183 & .003 & .018 & .859 & .000 & .120 & .003 \\
\hline$r r$ & 335 & .782 & .048 & .024 & .137 & .009 & .591 & .042 & .021 & .346 & .000 \\
\hline ru & 336 & .685 & .077 & .027 & .202 & .009 & .045 & .083 & .000 & .872 & .000 \\
\hline$r d$ & 335 & .701 & .075 & .036 & .179 & .009 & .322 & .555 & .000 & .033 & .090 \\
\hline$u c$ & 335 & .116 & .269 & .003 & .612 & .000 & .200 & .457 & .000 & .343 & .000 \\
\hline$u l$ & 336 & .062 & .345 & .000 & .592 & .000 & .021 & .872 & .003 & .101 & .003 \\
\hline$u r$ & 334 & .156 & .216 & .000 & .629 & .000 & .581 & .051 & .027 & .335 & .006 \\
\hline$u u$ & 334 & .084 & .260 & .000 & .656 & .000 & .033 & .108 & .000 & .859 & .000 \\
\hline$u d$ & 335 & .096 & .191 & .000 & .713 & .000 & .343 & .558 & .000 & .033 & .066 \\
\hline$d c$ & 335 & .337 & .478 & .000 & .006 & .179 & .242 & .460 & .000 & .296 & .003 \\
\hline$d l$ & 334 & .237 & .599 & .000 & .006 & .159 & .012 & .880 & .000 & .108 & .000 \\
\hline$d r$ & 336 & .312 & .449 & .000 & .009 & .229 & .589 & .054 & .027 & .330 & .000 \\
\hline$d u$ & 335 & .310 & .504 & .000 & .015 & .170 & .030 & .116 & .000 & .854 & .000 \\
\hline$d d$ & 335 & .346 & .451 & .000 & .006 & .197 & .370 & .549 & .000 & .012 & .069 \\
\hline
\end{tabular}


Table A2. Empirical estimates of marginal distributions for the conteNt-conteXt system in Fig. 3 for participant $\mathrm{P} 2$.

\begin{tabular}{|c|c|c|c|c|c|c|c|c|c|c|c|}
\hline \multirow{2}{*}{$\begin{array}{l}\mathrm{P} 2 \\
\text { Context }\end{array}$} & \multirow[b]{2}{*}{ Trials } & \multicolumn{5}{|c|}{ Left response } & \multicolumn{5}{|c|}{ Right response } \\
\hline & & Center & Left & Right & $\mathrm{Up}$ & Down & Center & Left & Right & $\mathrm{Up}$ & Down \\
\hline$c c$ & 336 & .616 & .062 & .039 & .226 & .057 & .560 & .062 & .164 & .202 & .012 \\
\hline$c l$ & 336 & .586 & .080 & .033 & .268 & .033 & .265 & .604 & .015 & .107 & .009 \\
\hline$c r$ & 336 & .586 & .045 & .062 & .259 & .048 & .185 & .000 & .720 & .071 & .024 \\
\hline$c u$ & 336 & .607 & .083 & .033 & .220 & .057 & .131 & .062 & .089 & .717 & .000 \\
\hline$c d$ & 336 & .580 & .054 & .024 & .304 & .039 & .348 & .033 & .086 & .024 & .509 \\
\hline$l c$ & 336 & .223 & .604 & .000 & .134 & .039 & .610 & .092 & .119 & .152 & .027 \\
\hline$l l$ & 336 & .214 & .583 & .003 & .164 & .036 & .274 & .548 & .021 & .134 & .024 \\
\hline$l r$ & 336 & .223 & .586 & .009 & .158 & .024 & .220 & .006 & .682 & .065 & .027 \\
\hline$l u$ & 336 & .310 & .527 & .000 & .128 & .036 & .149 & .042 & .089 & .720 & .000 \\
\hline$l d$ & 336 & .226 & .557 & .003 & .179 & .036 & .333 & .039 & .098 & .021 & .509 \\
\hline$r c$ & 336 & .339 & .003 & .443 & .176 & .039 & .548 & .086 & .158 & .173 & .036 \\
\hline$r l$ & 336 & .318 & .012 & .432 & .205 & .033 & .247 & .631 & .018 & .080 & .024 \\
\hline$r r$ & 336 & .310 & .003 & .429 & .229 & .030 & .140 & .018 & .696 & .116 & .030 \\
\hline ru & 336 & .336 & .000 & .467 & .158 & .039 & .170 & .033 & .074 & .720 & .003 \\
\hline$r d$ & 336 & .351 & .000 & .405 & .211 & .033 & .381 & .054 & .095 & .030 & .440 \\
\hline$u c$ & 336 & .146 & .018 & .015 & .818 & .003 & .646 & .048 & .134 & .137 & .036 \\
\hline$u l$ & 336 & .131 & .030 & .015 & .821 & .003 & .345 & .545 & .012 & .068 & .030 \\
\hline$u r$ & 336 & .146 & .030 & .006 & .815 & .003 & .235 & .000 & .688 & .057 & .021 \\
\hline$u u$ & 336 & .167 & .021 & .018 & .795 & .000 & .196 & .036 & .128 & .637 & .003 \\
\hline$u d$ & 336 & .137 & .015 & .009 & .836 & .003 & .390 & .024 & .068 & .015 & .503 \\
\hline$d c$ & 336 & .354 & .030 & .036 & .021 & .560 & .539 & .057 & .143 & .229 & .033 \\
\hline$d l$ & 336 & .366 & .039 & .024 & .018 & .554 & .220 & .583 & .006 & .158 & .033 \\
\hline$d r$ & 336 & .375 & .039 & .006 & .009 & .571 & .199 & .003 & .661 & .119 & .018 \\
\hline$d u$ & 336 & .393 & .018 & .033 & .021 & .536 & .122 & .027 & .065 & .786 & .000 \\
\hline$d d$ & 336 & .360 & .039 & .024 & .036 & .542 & .393 & .048 & .116 & .021 & .423 \\
\hline
\end{tabular}


Table A3. Empirical estimates of marginal distributions for the conteNt-conteXt system in Fig. 3 for participant P3.

\begin{tabular}{|c|c|c|c|c|c|c|c|c|c|c|c|}
\hline \multirow{2}{*}{$\begin{array}{l}\text { P3 } \\
\text { Context }\end{array}$} & \multirow[b]{2}{*}{ Trials } & \multicolumn{5}{|c|}{ Left response } & \multicolumn{5}{|c|}{ Right response } \\
\hline & & Center & Left & Right & Up & Down & Center & Left & Right & Up & Down \\
\hline$c c$ & 336 & .738 & .092 & .012 & .143 & .015 & .634 & .149 & .015 & .188 & .015 \\
\hline$c l$ & 337 & .801 & .053 & .030 & .110 & .006 & .027 & .955 & .000 & .018 & .000 \\
\hline $\mathrm{cr}$ & 336 & .762 & .098 & .021 & . 107 & .012 & .321 & .003 & .631 & .036 & .009 \\
\hline$c u$ & 336 & .768 & .086 & .033 & .098 & .015 & .128 & .060 & .000 & .812 & .000 \\
\hline$c d$ & 335 & .785 & .081 & .021 & 101 & .012 & .648 & .081 & .009 & .003 & .260 \\
\hline$l c$ & 337 & .056 & .935 & .000 & .009 & .000 & .700 & .104 & .039 & .151 & .006 \\
\hline$l l$ & 336 & .060 & .929 & .000 & .012 & .000 & .045 & .929 & .000 & .027 & .000 \\
\hline$l r$ & 337 & .053 & .929 & .000 & .015 & .003 & .288 & .000 & .680 & .033 & .000 \\
\hline$l u$ & 337 & .059 & .917 & .000 & .021 & .003 & .148 & .059 & .006 & .786 & .000 \\
\hline$l d$ & 336 & .051 & .938 & .000 & .012 & .000 & .676 & .054 & .015 & .006 & .250 \\
\hline$r c$ & 336 & .336 & .000 & .649 & .012 & .003 & .658 & .125 & .024 & .185 & .009 \\
\hline$r l$ & 337 & .335 & .009 & .635 & .021 & .000 & .027 & .935 & .000 & .039 & .000 \\
\hline$r r$ & 336 & .312 & .000 & .670 & .012 & .006 & .298 & .000 & .667 & .036 & .000 \\
\hline ru & 337 & .332 & .000 & .653 & .015 & .000 & .142 & .071 & .000 & .783 & .003 \\
\hline$r d$ & 336 & .280 & .000 & .699 & .015 & .006 & .658 & .074 & .021 & .012 & .235 \\
\hline$u c$ & 336 & .164 & .033 & .003 & .801 & .000 & .699 & .134 & .021 & .137 & .009 \\
\hline$u l$ & 336 & .143 & .065 & .003 & .789 & .000 & .042 & .943 & .000 & .012 & .003 \\
\hline$u r$ & 336 & .134 & .033 & .003 & .830 & .000 & .327 & .000 & .631 & .033 & .009 \\
\hline$u u$ & 336 & .202 & .033 & .003 & .762 & .000 & .164 & .062 & .000 & .774 & .000 \\
\hline$u d$ & 337 & .172 & .021 & .003 & .804 & .000 & .668 & .080 & .015 & .000 & .237 \\
\hline$d c$ & 335 & .603 & .021 & .012 & .000 & .364 & .618 & .137 & .009 & .230 & .006 \\
\hline$d l$ & 337 & .626 & .030 & .027 & .000 & .318 & .030 & .950 & .000 & .021 & .000 \\
\hline$d r$ & 337 & .644 & .030 & .021 & .000 & .306 & .329 & .000 & .635 & .033 & .003 \\
\hline$d u$ & 337 & .638 & .030 & .015 & .000 & .318 & .151 & .080 & .003 & .766 & .000 \\
\hline$d d$ & 336 & .619 & .039 & .012 & .003 & .327 & .708 & .068 & .009 & .006 & .208 \\
\hline
\end{tabular}

